\title{
Towards a Remote Launch Mechanism of TV Companion Applications Using iBeacon
}

\author{
Louay Bassbouss \\ Fraunhofer FOKUS \\ Kaiserin-Augusta-Allee 31 \\ 10589 Berlin, Germany \\ louay.bassbouss@fokus.fraunhofer.de
}

\author{
Görkem Güçlü \\ Fraunhofer FOKUS \\ Kaiserin-Augusta-Allee 31 \\ 10589 Berlin, Germany \\ goerkem.gueclue@fokus.fraunhofer.de
}

\author{
Stephan Steglich \\ Fraunhofer FOKUS \\ Kaiserin-Augusta-Allee 31 \\ 10589 Berlin, Germany \\ stephan.steglich@fokus.fraunhofer.de
}

\begin{abstract}
TV sets and companion devices (Smartphones, Tablets, etc.) have outgrown their original purpose and are now playing together an important role to offer the best user experience on multiple screens. However, the collaboration between TV and companion applications faces challenges that go beyond traditional single screen applications. These range from discovery, wake-up and pairing of devices, to application launch, communication, synchronization and adaptation to target device and screen size. In this position paper, we will limit ourselves to the Remote Launch aspect and introduce an idea for a new mechanism for launching companion applications using iBeacon technology.
\end{abstract}

Keywords: Second Screen, Multiscreen, Application Launch, iBeacon, Bluetooth Low Energy

\section{INTRODUCTION}

Almost every modern connected TV can be controlled with a smartphone app provided by the manufacturer. These apps range from simple remote control replacements, to fully featured media centers including PVR programming, interactive EPGs and video streaming from the TV to the smartphone. Furthermore, new generation of companion devices provide connectivity to large displays and give mobile applications the ability to mirror or extend the small screen of the mobile device.

However, today's standard application models are focused on single devices and screens. Multiscreen applications face new challenges such as discovery of devices, launch of applications on remote devices, synchronization, application to application communication, etc. New application development paradigms, concepts, protocols and technologies addressing these challenges are getting mature. However, there are still interoperability issues and limitations between different devices and platforms: for launching companion applications on Android and iOS devices e.g. from applications running on the TV, we need to consider the different capabilities and limitations of each platform. In this work, we will propose a mechanism for launching applications on remote devices using the iBeacon technology.

\section{TECHNICAL DISCUSSION AND CONCEPT'S IDEA}

The traditional way for launching applications on smart TVs is by using the TV remote control or a special mobile application provided by the TV manufacturer. To launch a TV application that collaborates with an application running on a companion device, e.g. YouTube application on TV for displaying videos and YouTube control application on companion device, additional steps are needed. Both applications need to be connected to the same session in order to know about each other and establish a communication channel. PIN or QR code technologies can help here. The application running on the TV displays a PIN or QR code. The user of the companion device can either scan the QR Code or enter the PIN code manually in the companion application and join the session. To reduce the number of steps needed to connect both apps to each other, Network Service Discovery (NSD) technologies like SSDP [1] or Bonjour [2] can help. The Discovery and Launch Protocol DIAL [3] is especially made for this purpose. TV sets that support this protocol implement the DIAL server functionality and advertise a DIAL service in the local network using SSDP. Companion devices implement the DIAL client functionality and discover DIAL devices available in the network. Once a DIAL device is found, the companion device connects to it and uses its service interface to launch a specific app. During Launch, the companion application can pass some parameters to the TV application such as session ID, video URL, etc.

Hybrid TV technologies like Hybrid Broadband Broadcast TV (HbbTV) [4] can also profit from the Remote Application Launch feature: Involving companion screens within Hybrid TV sessions opens the door to a new kind of use cases and offers a replacement of traditional remote control that is not suitable for the interaction with such smart TV services. The key to successful integration of TV services and companion devices is the seamless and user friendly notification and launch of companion applications. Many Hybrid TV services are using the PIN or QR code technology for this purpose to ensure cross-platform support since these technologies are not dependent from platform specific APIs. On the other side, studies showed that these technologies are more suitable for printed material and not for the digital domain from user experience perspective [5]. Technically, The DIAL technology can be used for launching companion applications by implementing the DIAL server functionality on companion devices and client functionality on TV, but this will raise additional security and battery lifetime issues on companion devices. Furthermore, this requires companion devices to run services in the background which is limited on some mobile platforms like iOS. In addition, the flow for remotely launching companion applications is not the same as for launching TV applications from the end-user perspective. Bringing an 
application on a companion device to the foreground without asking the user while he interacts with another application is a bad user experience. Most mobile platforms allow this feature only in relation with a user interaction e.g. through Notifications. On iOS, there are two types of notifications: local and remote notifications: they differ only in the way how they are triggered: local notifications are triggered by applications running in the background on the same device while remote notifications are triggered and sent by Apple Push Notifications Service (APNs). This means, if an application is not running at all (neither in the foreground nor in the background), the user can be notified only through remote notifications. One option to wake-up and launch a not running iOS application in the background is by using iBeacon [6], a new technology that extends Location Services in iOS7. iBeacon uses a Bluetooth Low Energy (BLE) [7] signal which iOS devices detect. Any device supporting BLE can be turned into an iBeacon transmitter and alert applications on iOS devices nearby. iBeacon transmitters are in general tiny and cheap sensors that can run many years with a single coin battery depending on how frequent they broadcast information around. Main usage area of iBeacon are location based services: iOS devices alert apps when the user approaches or leaves a Beacon's region. While a beacon is in range of an iOS device, apps can also monitor for the relative distance to the beacon. If the companion application was not running while the user crosses (enters or leaves) a Beacon's region, the iOS device wakes up the application and launches it in the background only for around 10 seconds (iOS limitation). The application can react in this time frame to changes of user's location and may show a local notification, through which the user can bring the application to the foreground. An iBeacon message consists besides the BLE packet headers and Apple's static prefix of a 128-bit 'proximity UUID' value that uniquely identifies one or more beacons as a certain type or from a certain organization, a 16-bit unsigned integer 'major' value that can be used to group related beacons that have the same proximity UUID and a 16-bit unsigned integer 'minor' value that differentiates beacons with the same proximity UUID and major value. iOS applications can use the iBeacon API in iOS for registering a Beacon's region using the proximity UUID. If a device crosses the boundaries of a registered beacon's region, the application will be notified on entering or leaving that region. Application providers need to choose a value for the proximity UUID and use it in all beacons as well as in the iOS application. This means, the proximity UUID is in general a fix value in the context of a specific application or organization.

iBeacon seems to be a promising technology not only for location based services, but also for launching companion applications in a multiscreen environment if new generation of TV sets are equipped with BLE sensors and act as beacon transmitters. Main advantage of this approach is that the TV will able to wake up companion applications only on devices belonging to users sitting in front of the TV, it doesn't matter if they are connected to local network or mobile carrier network. Unlike the traditional usage of iBeacon where the proximity UUID is fix and known for a specific application, a more dynamic behavior by using different values for proximity UUID on different TV sets is required in the multiscreen domain: If the $\mathrm{TV}$ manufacturer uses a unique proximity
UUID, the companion application will be always notified when the user crosses the beacon's region of any TV from the same manufacturer. In case TV sets transmit different proximity UUIDs, it will be possible to notify companion applications associated with a specific TV. Furthermore, it will be possible for a companion application to subscribe to different TV's beacon regions at the same time and therefore to get notified by different TV sets, e.g. if the user has more than one $\mathrm{TV}$ at home. Since there is no unique and known proximity UUID to be used in the TV companion application, we need a mechanism to generate and exchange proximity UUIDs between the TV and the companion application. Proximity UUIDs can be generated randomly and stored on the TV without any user interaction. The probability of collision with proximity UUIDs used in other applications is almost zero since proximity UUIDs are 128-bit long. The proximity UUID can be exchanged between the TV and companion devices during the setup phase of the TV's Companion Application. Companion devices can report their presence nearby the TV (in range or out of range) to a central presence server. The presence server notifies the TV each time the presence of a companion device changes. The TV manages a table of companion devices in its range and can send a request to the server to launch a companion application on a specific device. The server sends a push notification to the requested device that displays a launch message for the user. If the user clicks on the notification, the requested application will be launched and connected to the TV using the proximity UUID as session identifier.

\section{CONCLUSION}

We introduced in this paper a very general idea for a Remote Application Launch mechanism using iBeacon technology. We will introduce in next step more details about each component of the system and how these components collaborate with each other. We will also consider other aspects such as usability, privacy, security and performance and provide a proof-of-concept implementation.

\section{REFERENCES}

[1] Contributing Members of the UPnP Forum, UPnP Device Architecture 1.1, UPnP Forum Std., October 2008, [Section 1]. [Online]. Available: http://upnp.org/specs/arch/UPnP-arch-DeviceArchitecture-v1.1.pdf

[2] Apple - Bonjour, "Bonjour - apple support," 2014, [Online; accessed 4June-2014]. [Online]. Available: https://www.apple.com/support/bonjour/

[3] Netflix, DIscovery And Launch protocol specification, Version 1.7, Netflix Std., 2014. [Online]. Available: http://www.dial-multiscreen.org/dialprotocol-specification/DIAL-2ndScreenProtocol-1.7.pdf

[4] HbbTV association, "Hbbtv," 2014, [Online; accessed 4-June-2014]. [Online]. Available: https://hbbtv.org/

[5] eMarketer, "Us ahead of western europe in qr code usage. consumers most familiar with qr codes on magazines, printed materials." 2014, [Online; accessed 4-June-2014]. [Online]. Available: http://www.emarketer.com/Article/US-Ahead-of-Western-EuropeQR-Code-Usage/1009631

[6] Apple - iBeacon, “ios: Understanding ibeacon," 2014, [Online; accessed 4-June-2014]. [Online]. Available: http://support.apple.com/kb/HT6048

[7] Bluetooth SIG, "Bluetooth smart technology: Powering the internet of things," 2014, [Online; accessed 4-June-2014]. [Online]. Available: http://www.bluetooth.com/pages/bluetooth-smart.aspx 